\title{
Gabriele Czarnowski \\ Kreuzung als Experiment. Beziehungen zwischen Genetik und staatlichem Gesundheitswesen im Nationalsozialismus
}

Zusammenfassung: Dieser Beitrag thematisiert das Verhältnis von wissenschaftlicher Rationalität und staatlicher Bürokratie in der Geburten-und Rassenpolitik des Nationalsozialismus. Unter Berufung auf Wissenschaft erließ der nationalsozialistische StaatGesetze, die massive Eingriffe in die Freiheit und Unverletzbarkeitder Person beinhalteten, zugleich errichtete er den dafür notwendigen Verwaltungsapparat. Die gesundheitliche, eugenische und rassistische Selektion war nur möglich durch die Allianz von Wissenschaft und Politik. Diese Allianz soll im folgenden nicht als Frage nach Bündnissen zwischen Personen behandelt werden, sondern am Aufweis struktureller Analogien zwischen genetischer Forschung, staatlicher Bürokratie und Praxis der Selektion. Damit reicht sie - wenn auch unter anderen Bedingungen - über die Zeit des Nationalsozialismus hinaus. Focus ist das die moderne Vererbungstheorie konstituierende Experiment der "Kreuzung".

\section{Eine neue wissenschaftliche Körper- und Gesellschaftspolitik: Rassenhygiene und Eugenik}

Als der nationalsozialistische Staat die Rassenhygiene zur Staatsdoktrin erhob, knüpfte er an eine moderne, internationale Entwicklung in Wissenschaft und Politik an, deren Geschichte durch eine Reihe von Untersuchungen inzwischen gut dokumentiert ist (Bergmann 1988, Bock 1986, Faith Weiss 1987, Gordon 1977, Graham 1977, Labisch/Tennstedt 1985, Macnicol 1989, Weindling 1984, 1985, Weingart/ Kroll/Bayertz 1988). Sie machen deutlich, daß Eugenik und die in Deutschland zunehmend antisemitisch ausgerichtete Rassenhygiene nicht allein als bloße Vorläufer des Nationalsozialismus betrachtet werden können, sondern daß hier ein fundamentales politisches, soziales und wissenschaftliches Glaubens- und Handlungssystem der Moderne entstand, dessen Ende und dessen Folgen noch nicht abzusehen sind. Im nationalsozialistischen Deutschland erfuhr es eine besonders brutale Konkretion. Rassenhygienische und eugenische Deutungsmuster sozialer Phänomene waren um die Jahrhundertwende Angelegenheit einer kleinen Gruppe von (vornehmlich Natur-) Wissenschaftlern und Ärzten, die sich in Deutschland ab 1905 in der Gesellschaft für Rassenhygiene organisierten. Bis Anfang der dreißiger Jahre konnten sie im öffentlichen, gesundheitspolitischen wie berufspolitischen Diskurs, an den Universitäten und in der Politikberatung beachtlichen Einfluß gewinnen. Eugenisches Denken fand Anhänger und Anhängerinnen in vielen gesellschaftlichen Gruppen, quer durch 
das Spektrum der politischen Parteien, in den Kirchen, bei der Linken, in der Frauenbewegung, war also kein Reservat der Konservativen oder der nationalen Rechten. Besonders verbreitet war es - außer in den Human- und Bio-Wissenschaften - unter Angehörigen von Berufsgruppen, die mit Menschen zu tun hatten (Grossmann 1987). Der gemeinsame Nenner für die breite Allianz in Fragen der Eugenik von Personen und Organisationen ansonsten höchst divergierender Anschauungen war neben ihrer Zukunftsorientiertheit vor allem ihr wissenschaftlicher und damit parteipolitisch neutraler Charakter. Eugenik als angewandte Wissenschaft entwickelte auf naturwissenschaftlicher Grundlage Strategien zur Lösung sozialer Probleme und galt von daher als sachlich, objektiv und fern von allem »Parteiengezänk «. Gleichwohl war sie nicht »unpolitisch «, sondern zur Umsetzung ihrer Programme auf die Gesetzgebungskompetenz und die finanziellen Ressourcen des Staates angewiesen.

Gegenstand der Rassenhygiene wie der Eugenik war nach Alfred Ploetz (1860-1940), dem »Altmeister « der deutschen Rassenhygiene, die Vitalrasse. Darunter faßte er im Gegensatz zur Systemrasse der messenden und beschreibenden Anthropologie (Ethnie) den durch »Fortpflanzung « gegebenen Zusammenhang der Gesellschaftsmitglieder. Der Rassenbegriff implizierte bei einer Reihe von Rassenhygienikern auch ein hierarchisches ethnisches Rassenverständnis. So setzten etwa Eugen Fischer (1874-1967), Fritz Lenz (1887-1976) oder Otmar von Verschuer (1896-1969) die »nordische Rasse « als Hort von Geist und Kultur an die Spitze aller »Rassen«. Um sich davon abzugrenzen, plädierte der Sozialhygieniker Alfred Grotjahn (1869-1931) für den Begriff der »Fortpflanzungshygiene«. Er schlug vor, den Terminus »Rasse « durch »Konnubialkreis « zu ersetzen oder durch »Konglomerat generativ untereinander verbundener Menschen « (Grotjahn 1926, S. 21 f.). Auch die Umbenennung der Deutschen Gesellschaft für Rassenhygiene in Deutsche Gesellschaft für Rassenhygiene (Eugenik) im Jahre 1931 beruhte auf Abgrenzungsabsichten gegenüber den ethnisch-rassistischen »Norden«-Anhängern und dem gegen Ende der Weimarer Zeit wachsenden Antisemitismus. Über die Entwicklung der Anthropologie zur AnthropoBiologie - eine Vorläuferin der heutigen Populationsgenetik - wurde die theoretische Verbindung von anthropologischem und hygienischem bzw. eugenischem Rassenbegriff hergestellt: Die Anthropo-Biologie definierte Rassenunterschiede als Erbunterschiede. Hierbei spielten vor allem Eugen Fischer und das 1927 gegründete erste deutsche humangenetische Institut, das Kaiser Wilhelm-Institut für Anthropologie, menschliche Erblehre und Eugenik in Berlin-Dahlem eine maßgebende Rolle (Bergmann/Czarnowski/Ehmann 1989). Trotz beträchtlicher Differenzen stimmten Rassenhygiene, Eugenik und Fortpflanzungshygiene in dem Objekt ihres Interesses überein: »Fortpflanzung «. Außerdem beurteilten sie den Menschen nach seinem sozialen Wert; von »Minderwertigen « und »Höherwertigen «, »Aszozialen « und »gesunden Familien« zu sprechen, gehörte schon in den zwanziger Jahren zum gängigen öffentlichen wie professionellen Sprachgebrauch. In der Überzeugung, daß die »Beschaffenheit « jedes Menschen neben Umwelteinflüssen wesentlich von seinen »erblich überkommenen Anlagen« (Grotjahn 1926, S. III) bestimmt werde, zogen sie die Schlußfolgerung, daß Sexualität und »Fortpflanzung « nicht (mehr) dem Zufall oder 
dem Gutdünken der individuellen Menschen oder der (Ehe-) Paare allein überlassen bleiben könne, sondern der Beratung, Überwachung und regulierender Eingriffe durch Experten bedürfe. Zu berufenen »Sach«-Verständigen für diese Tätigkeit in Forschung und Praxis erklärten Rassenhygieniker und Eugeniker ihre eigene Zunft: Naturwissenschaftler und Ärzte.

Nach Ende des ersten Weltkrieges mit den vielen Kriegstoten, zerrütteten Ehen und dem von Politikern und Demographen beklagten »Geburtenausfall«glaubte auch die Politik, nicht mehrohne diese Experten auskommen zu können. Damit wurde der zuvor rechtlich streng der Privatheit der Familie und des Arzt-Patienten-Verhältnisses zugeordnete Bereich ehelicher Sexualität, Zeugung und Geburt politisch zum wissenschaftlich normierungsbedürftigen Handlungsbereich gemacht. Diese Entwicklung war zugleich Ausdruck eines sich vollziehenden fundamentalen gesellschaftlichen Wandels des sozialen Geschlechterverhältnisses und der Moralvorstellungen, was hier nicht weiter erläutert werden kann. Mit der Etablierung eines Beirats für Rassenhygiene beim Preußischen Minister für Volkswohlfahrt - nach zwei Jahren wurde er als Ausschuß für BevölkerungspolitikundRassenhygiene im neu gegründeten Preußischen Landesgesundheitsrat weitergeführt - hielten Rassenhygiene und Eugenik offiziell ihren Einzug in die wissenschaftliche Politikberatung. Als Mitglieder dieses Beirats wurden hauptsächlich Genetiker und Mediziner berufen (Bergmann 1988, Weindling 1984). Alle einschlägigen geburtenpolitischen Themen (Eheberatung, Verhütungsmittel, Abtreibung, Sterilisation, Elternschaftsversicherung) wurden hier verhandelt. Resultat der Arbeit waren Gesetzesvorstöße für obligatorische ärztliche Ehezeugnisse (1922), die Zulassung von Indikationen für Schwangerschaftsunterbrechung (1925/26), Sterilisation (1932) sowie die Schaffung neuer sozial- und geburtenpolitischer Infrastrukturen (Eheberatungsstellen 1927) (Grossmann 1984, Usborne 1989). Die Gesetzesentwürfe konnten allerdings nie die notwendige Reichstagsmehrheit auf sich vereinigen. Eine im Sinne der Rassenhygiene erfolgversprechende Politik konnte nur unter den Bedingungen der Diktatur realisiert werden - eine Tatsache, der sich die deutschen Rassenhygieniker bewußt waren. Ein Zitat aus der Schlußansprache Eugen Fischers auf dem internationalen Kongreß für Bevölkerungswissenschaft 1935 in Berlin, auf dem die deutschen Zwangssterilisationen erhebliche Diskussionen und Proteste ausgelöst hatten, kann dies illustrieren: »Die nationalsozialistische Führeridee hat es fertiggebracht, eine Frage, die sonst noch jahrelang Kommissionen oder Parlamentsausschüsse und Sitzungen beschäftigt hätte, im vollen Gefühl persönlicher eigener Verantwortung durch die Tat eines einzelnen Mannes zu lösen. Nichts hat die Frage selbst, sei die Lösung wie sie wolle, so gefördert, wie diese Tat.

Nie hat bisher eine rein wissenschaftliche Lehre, in diesem Fall die Erblehre, bewußt eine so große Verantwortung für die Zukunft der Menschheit auf sich genommen. Es ist ein Wendepunkt in der Geistesgeschichte der Menschheit, daß sie heute naturwissenschaftlich medizinische Erkenntnis nicht mehr nur individuell und je nach Wollen und Wunsch zur Erhaltung des Lebens des Einzelmenschen anwendet, sondern als Waffe und Richtschnur dem Staatsmann zum Wohle seines Volkes mit wohltätigem Zwang an die Hand gibt« (Fischer 1936).

Der Unterschied zwischen der Weimarer Republik und dem »Dritten Reich « liegt also weniger in den Begründungen des Zugriffs auf Körper und (»Privat «-) Leben als in der legalen wie illegalen Gewalt, mit der er gegen das Grundrecht der Freiheit und körperlichen Unversehrtheit der Person durchgesetzt wurde. 


\section{Kreuzen und Quantifizieren: Sexualität und $»$ Fortpflanzung« als naturwissenschaftliches Experiment}

Die naturwissenschaftliche Grundlage der Eugenik und Rassenhygiene bildete neben der Darwinschen Selektionstheorie die Vererbungslehre. Die Allgemeine Vererbungslehre oder Experimentelle Genetik war eine noch junge Wissenschaft, die, zunächst angesiedelt in der Botanik und Zoologie, seit der Wiederentdeckung der Mendelschen Gesetze um 1900 einen rapiden Aufschwung nahm und im Zuge der breiten Begeisterung für rationale Welterklärung auch die Frage nach der Vererbung beim Menschen neu stellte. Bei Rassenhygienikern und Eugenikern fand die sich entwikkelnde Genetik große Aufmerksamkeit, bildeten doch Vererbung und Auslese den zentralen Mechanismus rassenhygienischer Gesellschaftstheorie. Die moderne Genetik wurde in zweierlei Hinsicht bedeutsam. Wissenschaftshistorisch hatte sie eine außerordentlich innovative Funktion für die medizinische und psychiatrische Vererbungsforschung - also die Forschung über Erbkrankheiten, die später die Indikationen für Zwangssterilisation und Eheverbote im Nationalsozialismus abgeben würden. Auf sozialpolitischer Ebene konnte sie eine außerordentlich weitreichende Wirkungsmacht entfalten: Sie verhalf der Rassenhygiene zum Status einer fundierteren naturwissenschaftlichen Gesellschaftswissenschaft, die geburtenpolitisches Eingreifen theoretisch als dringend erforderlich begründen konnte, wenn nicht bestimmte »Varianten « der Spezies Mensch unwiederbringlich verloren gehen sollten.

Gregor Mendel hatte 1865 bei Inzuchtversuchen mit rund- und kantigsamigen Erbsen herausgefunden, daß selbständige und konstante Einheiten, die »Erbeinheiten « (Gene), die Grundlage der Vererbung von bestimmten »Erbeigenschaften « sein müßten, da Merkmale, die in der ersten Generation verschwanden, in der zweiten und den folgenden wieder auftauchten und zwar in einem konstanten Zahlenverhältnis, das der binomischen Formel entsprach. Die älteren Vererbungsvorstellungen hingegen gingen davon aus, daß alle Merkmale »vermischt« und bei »Kreuzung « verschiedener »Rassen« verdünnt weitergegeben werden. Tier- und Pflanzenzüchter hatten zwar bereits vor Mendel in Experimenten die Vererbung vieler Merkmalsunterschiede festgestellt, aber sie trennten sie nicht voneinander und zählten sie nicht einzeln aus. Das Einmalige bei Mendel war - so der heutige Genetiker Monroe Strickberger - $\gg$ daß er jedes Merkmal getrennt betrachtete, daß er die Häufigkeit des Auftretens der verschiedenen Merkmale bei den Individuen in jeder Generation feststellte und daß er seine Zahlenergebnisse als Verhältnisse analysierte, die die zugrundeliegenden GesetzmäBigkeiten der Vererbung ausdrückten « (Strickberger 1988, 104).

Die Genetik trat somit in den Kreis der »exakten Wissenschaften « aufgrund der Methoden, mit denen sie arbeitete: das Experiment und die Statistik. Sie bildete sich nicht nur langsam zur eigenständigen Wissenschaft aus, sondern hatte auch Einfluß auf die medizinisch-psychiatrische Vererbungsforschung. Das spezifische und zugleich die moderne Genetik konstituierende Experiment ist die »Kreuzung «. Über den Zusammenhang von allgemeiner Genetik und menschlicher Erbforschung heißt es in dem ersten systematischen humangenetischen Lehrbuch in Deutschland: In 
ihren allgemeinen Sätzen gründe sich die menschliche Erblehre auf Ergebnisse der experimentellen Erbforschung an Tieren und Pflanzen, in ihren speziellen Sätzen auf direkte Erfahrungen am Menschen. Während jedoch der experimentierende Erbforscher bestimmte Kreuzungen absichtlich herbeiführe, suche der menschliche Erbforscher sie auf: »Alle Kreuzungen sind schon irgendwo vorhanden « (Baur/Fischer/ Lenz 1936, S. 593). Jeder Mensch erscheint als »Kreuzungsprodukt« seiner Eltern. In seinen Kindern, Geschwistern, Großeltern, Onkeln, Tanten, Neffen und Nichten spiegelt sich gleichsam retrospektiv das Vererbungsgesetz hinsichtlich bestimmter Merkmalsverteilungen, hergestellt durch »Fortpflanzung « und »Vererbung« als Weitergabe von Genen. ${ }^{1}$

$\mathrm{Da} \beta$ die allgemeine Genetik ihre Ergebnisse durch Kreuzungsexperimente an Pflanzen und Tieren gewann, stand der Annahme der prinzipiellen Gültigkeit der Vererbungsgesetze nicht entgegen, davon gingen die Wissenschaftler aus. Grundsätzlich war der einheitliche Blick auf Pflanze, Tier und Mensch möglich, seitdem die moderne Botanik, Zoologie und Medizin die Zelle als Grundbaustein aller Lebewesen definierten, insbesondere die spezifische Organisation und Bedeutung der Keimzellen und der Chromosomen. Die gleiche Vererbungsgesetzmäßigkeit anzunehmen, war und ist unter naturwissenschaftlicher Perspektive durchaus logisch, auch wenn die Vorgänge im einzelnen nicht bekannt waren. Sie auf Basis der neuen Methode zu finden, war eine Herausforderung an die Forschung und eine Frage der Zeit. Die sich entwickelnde menschliche Erbforschung folgte den experimentell gewonnenen Gesetzen der Allgemeinen Genetik (Roth 1986, Weß 1989).

Die Zugehörigkeit der modernen Genetik und in ihrer Nachfolge auch der Humangenetik zu den Naturwissenschaften begründet sich also durch die Quantifizierung und das Experiment. Die Genetik führte Wahrscheinlichkeitsrechnung und neue statistische Verfahren auf Basis ausgedehnter Familien- und seit etwa Mitte der zwanziger Jahre der Zwillingsforschung als Mittel zur Erklärung bestimmter Krankheitsursachen in Medizin und Psychiatrie ein. Aussagen über die Ursachen von Qualitäten wurden durch Berechnungen über die Häufigkeit ihres familialen Vorkommens ersetzt bzw. ergänzt, also durch an Sexualität und Geburt gebundene Quantitäten. Was das für die Psychiatrie bedeutete, die seit Mitte des 19. Jahrhunderts fast alle $\gg$ Geisteskrankheiten« als von den Eltern ererbt ansah, soll weiter unten andiskutiert werden.

\section{Die "Beständigkeit des Erbguts" als Basis einer naturwissenschaftlichen Gesellschaftstheorie}

Die von Mendel gefundene Konstanz in der Vererbung einzelner Merkmale und ihre Quantifizierung machten es möglich, den bis dahin zumeist individuell oder familial verstandenen Begriff der Entartung oder Degeneration als Erklärung für soziale Prozesse auf die Gesellschaft als Ganzes zu beziehen. Den Entartungsbegriff hatten die Rassenhygiene und Eugenik aus der Degenerationslehre der Psychiatrie übernommen (Bergmann 1988), die damit in der Tradition des französischen Psychiaters Auguste Morel stand. Als »entartet« diagnostizierten die Psychiater der zweiten Hälfte 
des 19. Jahrhunderts global Menschen mit bestimmten »Abnormitäten«, ihre Eltern und Großeltern. Die »Krankheit « galt als progredient: Mit ihr sei eine allgemeine Verschlechterung der Familien von Generation zu Generation verbunden. Die moderne Genetik veränderte den Entartungsbegriff. Sie hob die Vorstellung von Entartung als einem »unentrinnbar fortschreitenden Verhängnis « auf und setzte an ihre Stelle eine quantitative Definition von Entartung als »Neuentstehung und Ausbreitung krankhafter Erbanlagen«. In demselben $\mathrm{Maß}$ aber, wie sie das schreckliche, auf Generationen von Familien lastende Verhängnis stoppte, machte sie auch der Vorstellung von einer unbegrenzten individuellen Höherentwicklung den Garaus. In beide Richtungen schränkte die neue Sichtweise ein: sie legte die Entwicklungsvoraussetzungen in jeden einzelnen Menschen selbst und in seine Erbanlagen bzw. Gene. Dies ist insofern beachtenswert, als in jener Zeit kulturpessimistische Strömungen eine bedeutende Rolle spielten. Der moderne Entartungsbegriff war nicht mehr ein qualitativer Sammelbegriff für verschiedenste körperliche, soziale, sittliche oder charakterliche Degenerationen innerhalb einer Familie, sondern er drückte ein durch Vererbung und »Fortpflanzung « konstituiertes gesellschaftliches Mengenverhältnis aus. Der Sozialhygieniker Alfred Grotjahn erklärte:

»Die allgemeine Entartung ist vorwiegend als ein quantitativer Vorgang aufzufassen, der dadurch zustande kommen kann, daß die minderwertigen Glieder sich dauernd stärker fortpflanzen als die Rüstigen und diese schließlich vollständig überwuchert werden.«(Grotjahn 1926, 15 f.)

Der quantitative Entartungsbegriff ließ die Gesellschaft als System kommunizierender Röhren erscheinen: »Höherwertige« und »minderwertige Erblinien« (Familien) wurden miteinander verbunden und in ein bestimmtes generativ-statistisches Verhältnis gesetzt. Berühmt sind die Schaubilder, auf denen die Kinderzahlen von Schwarzen und Weißen in Südafrika generationenweise für $300 \mathrm{Jahre} \mathrm{hochgerechnet} \mathrm{werden.} \mathrm{Sie}$ sollten ebenso wie Schaubilder über die Geschwisterzahlen von »Begabten« und »Unbegabten « die »drohende Entartung « belegen.

Die durch die moderne Genetik mit einem festeren Fundament versehene Rassenhygiene (Eugenik) definierte Rassen, Klassen und Geschlechter als Gruppen vorhandener $\gg$ Erbmassen« (gen pools) von unterschiedlicher Beschaffenheit oder Qualität. Der Qualitätsbegriff war - von Lenz offen gekennzeichnet - ein Wertbegriff. Als Maßstab galt ein bestimmter Standard von Kulturbegabung, den er als erbliche Veranlagung zu gelingender oder nicht möglicher Anpassung an die Erfordernisse der modernen Kultur formulierte (Lenz 1932, S. 11). Fortpflanzung wurde so zu einem wesentlichen Faktor für die Vermehrung, Erhaltung oder das Verschwinden des jeweiligen Vorrats oder stocks an »verschieden wertvollem Erbgut«. Der Pflanzengenetiker Erwin Baur (1930, S. 429) sah dies so:

»Wir können ... mit großer Wahrscheinlichkeit heute damit rechnen, daß, wie bei jeder allogamen Spezies, so auch beim Menschen die serbliche Variation< im wesentlichen auf der bei jedem Sexualakt stattfindenden Neukombination mendelnder Faktoren beruht. Was wir ein >Volk $<$ heißen, etwa die >Deutschen $<$, ist ... ein buntes mendelndes Gemisch, das entstanden ist aus der Kreuzung einer ganzen Reihe (auch in sich ursprünglich nicht einheitlichen) $>$ Rassen<.

Ein solches Gemisch bleibt bei freier Panmixie ... in seiner bunten Zusammensetzung konstant, falls kein Selektionsprozeß eingreift. Sowie aber eine Selektion durch bestimmte herausmendelnde Typen erfolgt, muß sich, wenn auch langsam, die durchschnittliche Zusammensetzung ändern.« 
Unter dieser Perspektive erhielten Ehelosigkeit, Heiratsalter, Kinderzahl bzw. Kinderlosigkeit, männliches und weibliches vor- und außereheliches Sexualverhalten (Abtreibung, Antikonzeptiva, Sterilisation) der verschiedenen Ethnien und Klassen eine ungeheure Wichtigkeit als »Ausleseinstrument «: Sie regulieren die weitere Entwicklung der unterschiedlichen Erbmassen und ihr Verhältnis zueinander. Differenzierte Fortpflanzung war der Oberbegriff, unter dem die Eugenik hier »sozialbiologische « (familienstatistische) Erhebungen betrieb und feststellte, daß »das Erhaltungsminimum nur bei den ungelernten Arbeitern überschritten« und »in verhältnismäßig wenigen Generationen ... also jener körperlich und seelische Typus, den heute die ungelernten Arbeiter aufweisen, den Typus der ganzen Bevölkerung darstellen« werde. Die Gegenauslese schreite also weiter fort (Lenz, 1932, S. 154). Die neue genetische Vererbungstheorie spielte für die Begründung eine entscheidende Rolle: »Solange man glaubte, daß ein ... fortdauernder Ausleseprozeß, der gerade die besten >Varianten ausmerzt, die Variationsmöglichkeiten des Ausgangsmateriales nicht ändert, konnte man unbesorgt sein, man sah in den sozial tiefer stehenden Schichten und in der Landbevölkerung eine unversiegbare Quelle, aus der immer wieder genügendes Material von führenden Elementen hervorgeht«, führte Erwin Baur in seiner Vorlesung aus. Diese Anschauung sei aber unbedingt falsch:

Wenn »die Variation im wesentlichen auf Neukombination mendelnder Unterschiede« beruhe, werde »eine dauemde Ausmerzung bestimmter Typen, d.h. bestimmter Kombinationen sehr rasch zur Veränderung des Ausgangsmateriales führen. Wenn nur einige hundert Jahre lang gerade die besten Elemente aus dem Volk ausgelesen und an der geniigenden Fortpflanzung gehindert werden, dann wird das Volk im Durchschnitt rasch schlechter, wird vor allem nicht mehr die zur Erhaltung seiner Kulturstufe nötige Anzahl tüchtiger führender Elemente hervorbringen« (Baur 1930, S. 431).

Hier riefen Rassenhygieniker und Eugeniker den Staat auf, überwachend und regulierend einzugreifen.

\section{Mendelistisch-genealogische Erbforschung in der Psychiatrie}

Die Psychiatrie »vor Mendel« ging von einer ungeschiedenen, polymorphen Vererbung der Geisteskrankheiten aus, obwohl seit 1824 versucht wurde, sie massenstatistisch zu erklären. Die Massenstatistik der psychiatrischen Erbforschung im 19. Jahrhundert hatte das zahlenmäßige Verhältnis der Gesunden zu den Kranken in den einzelnen Familien nicht berücksichtigt, sie hatte alle Arten von Geistesstörungen oder Abweichungen zusammengerechnet, ohne eine "Scheidung der belastenden Momente nach klinischen Entitäten« vorzunehmen und hatte die Frage des Erbmodus nicht bedacht (Entres 1928, S. 52). Der große Neuerer war der Psychiater Ernst Rüdin (1874-1952), Leiter der genealogisch-demographischen Abteilung der durch Kraepelin gegründeten Deutschen Forschungsanstalt für Psychiatrie in München, die 1924 ein Institut der Kaiser Wilhelm-Gesellschaft wurde. Rüdin war Verfechter der Sterilisation »Minderwertiger « seit 1903 und einer der Mitverfasser des amtlichen Kommentars zum Gesetz zur Verhütung erbkranken Nachwuchses im Nationalsozialismus. Seine Studic Zur Vererbung und Neuentstehung der Dementia Praecox von 
1916 galt als »erstes Werk der streng mendelistisch-genealogischen Erbforschung in der Psychiatrie, die hinsichtlich der mathematisch-statistischen Auswertung des Ergebnisses « unanfechtbar sei (Entres 1928, S. 123 ff.). Rüdin hatte, ausgehend von »klinisch klaren Fällen« schizophrener »Probanden« über 700 Personen aufgesucht und Eltern, Geschwister, Stiefgeschwister und Kinder auf das Vorkommen von Schizophrenie und »andersartigen psychotischen Erkrankungen« klassifiziert. Aufgrund von Rechenoperationen entsprechend der mendelschen Statistik kam er zu Wahrscheinlichkeitsaussagen über den Erbgang, die die Erblichkeit der Schizophrenie beweisen sollten.

Das grundlegende Problem besteht nicht darin, daß gezählt wurde, sondern was gezählt wurde. Ob verschiedenförmige Erbsen oder ein bestimmtes klassifiziertes abweichendes Verhalten gezählt wird, ist vom Zählen selbst unabhängig. Die menschlichen Erbforscher griffen auf die vorhandene klinische Diagnostik zurück. Für die psychiatrische Erbforschung tat sich hier jedoch ein Berg von Problemen auf: »Im Bereich des Psychisch-Pathologischen haben Erblichkeitsuntersuchungen mit einer Hauptschwierigkeit zu rechnen «, führte der Psychiater Lothar Entres im Handbuch der Geisteskrankheiten 1928 aus, denn »die Krankheitsformen, zu deren Aufstellung die klinische Psychiatrie dank der Initiative Kraepelins gekommen ist, sind nicht nur hinsichtlich deren Grenzfestsetzung, sondern auch in bezug auf ihre reale Existenz heiß umstritten«. Sie begebe sich auf schwankenden Boden, wenn sie die heute wohl ausschließlich anwendbare Kraepelinsche Systematik der Geisteskrankheiten ihren Arbeiten zugrundelege: »Das größte Hindernis für eine unangreifbare und endgültige Einteilung der Psychosen liegt in unserer Unkenntnis der bewirkenden Hauptursachen bei den meisten Krankheitsformen. « Der Praktiker halte es am besten mit Karl Jaspers, der einerseits unumwunden zugebe, daß die Psychiatrie zur Zeit über einwandfreie Krankheitsbegriffe nicht verfüge, andererseits aber entschieden die für den Empiriker zwingende Notwendigkeit betone, $\gg$ mit dem provisorischen Krankheitsbegriff wie mit einer Fiktion zu arbeiten « (Entres 1928, S. 110 ff.). Die rüdinsche Untersuchung und andere »streng mendelistisch « arbeitenden Erbforscher hätten jedoch mit diesen Problemen praktisch wenig zu schaffen, weil sie von »Probanden« mit »klaren Diagnosen« ausgingen. Mögen hier noch »eindeutige Fälle« vorgelegen haben - die ihrerseits eine Übereinkunft von Psychiatern darstellen - so war dies spätestens bei der Untersuchung und Beurteilung der Familienmitglieder, was zur Berechnung des Erbgangs theoretisch ja zwingend war, nicht mehr der Fall. Zur Berechnungsgrundlage wurden - und werden - letztlich völlig willkürliche Beobachtungen und summarische Klassifizierungen des Forschers gemacht. Der heutige Außenseiter-Psychiater und Psychiatrie-Kritiker Thomas Szasz beurteilt sie schlicht als »erbärmlichen Humbug « (1979, S. 114). Er zitiert aus einer Untersuchung des »Gründungsvaters der modernen psychiatrischen Genetik « in den USA, Franz J. Kallmann aus dem Jahre 1938. Kallmann hatte Verbindungen zu Rüdin und war zu Gastaufenthalten an seinem Institut gewesen. Als Jude wurde er aus Deutschland vertrieben. Seine Studie Erbprognose und Fruchtbarkeit bei den verschiedenen Formen der Schizophrenie von 1935 allerdings ist im Sterilisationsgesetzkommentar 1936 aufge- 
führt. Noch auf dem bevölkerungswissenschaftlichen Kongreß 1935 hielt er es für »wünschenswert, die Fortpflanzungsverhinderung auch auf die auffälligen Blutsverwandten der Schizophrenen auszudehnen und sie vor allem für sämtliche eugenisch unerwünschte Personen in den Anfang der Fruchtbarkeitsperiode zu legen« (vgl. Müller-Hill 1984, S. 14,33, 132). Er setzte seine Arbeiten in den USA fort. Nach dem Krieg war er Rüdins Entlastungszeuge im Spruchkammerverfahren. Kallmann schrieb:

»Bei der Klassifizierung der schizoiden Psychopathen interpretierten wir die Diagnose wieder so strikt wie möglich (sic, Szasz) ... Unser Begriff des Schizoiden Psychopathen umfaßt somit die ungeselligen, kalten, unentschlossenen und fanatischen Typen, welche Schneider als Prototypen katatoider, heboider, schizoider respektive paranoider Fälle ansieht, sowie Hoffmanns eigensinnige Dummköpfe, bösartige Tyrannen, verschrobene Spinner, überpedantische Plänemacher, gehemmte $>$ Musterknaben< und Tagträumer ohne Verbindung zur Realität.

Wir bezogen jedoch nur solchepsychopathischen Individuen mit ein, welche die fundamentalen schizoiden Charakteristika autistischer Introversion, emotionaler Unzulässigkeit, plötzlicher Temperamentsausbrüche und unangemessener motorischer Reaktionen auf emotionale Stimuli aufwiesen und bei welchen Symptome schizoider Abnormität wie Bigotterie, Frömmelei, Habgier, Aberglaube, Mißtrauen, Halsstarrigkeit oder'Aufsässigkeit in auffallendem oder unverhältnismäßigem Grad vorhanden waren bzw. die Persönlichkeit der Betreffenden beherrschten.«

Die in der genealogisch-statistischen Erbforschung gewonnenen Aussagen über »Manifestationshäufigkeiten« von »Geisteskrankheiten« wurden zur wissenschaftlichen Grundlage der Mehrzahl aller Sterilisationsentscheidungen im Nationalsozialismus gemacht, an denen Psychiater als Erbgesundheitsrichter mitwirkten. Besonders im Bereich der psychiatrischen Diagnostik, die rund $90 \%$ aller Sterilisationsentscheidungen im Nationalsozialismus begründen sollte (Bock 1986), wird deutlich, in wie hohem Maße die Verbindung von professioneller Definitionsmacht mit der vom Staat verliehenen Rechtskraft heillose und willkürliche Folgen haben mußte. Vor allem vermittels der Sterilisationsindikationen angeborener Schwachsinn und Schizophrenie und der Eheverbotsgründe Psychopathie und geistige Störung wurden bewußt und gewollt Tür und Tor geöffnet, um jedes dem Amtsarzt oder den Sterilisationsrichtern negativ auffallende soziale abweichende Verhalten, unter Umständen unter Einbeziehung eines fachpsychiatrischen Gutachtens, in eine Zwangssterilisation bzw. ein Eheverbot zu verwandeln, vorausgesetzt, sie beherrschten die Fachsprache und kannten die Diagnosetabelle. Die Professionalität und der damit verbundene soziale und rechtliche Status der Selekteure waren es auch primär, die Einwendungen von Betroffenen, die über beides nicht verfügten, zu untergeordneten Einwürfen machten, die aufzunehmen oder fallenzulassen allein den entscheidenden Experten zustand. Die Betroffenen hatten keinerlei Recht, selbst auf die Diagnostik Einfluß zu nehmen. Über diese konnte sich ein Disput auf gleicher Rangstufe nur unter Experten entwickeln; ein solcher Disput konnte unter Umständen möglicherweise positive Folgen für Betroffene haben. Ab 1936 wurden zentrale Anweisungen an Sterilisationsrichter und Amtsärzte erlassen, auf die Einwände derjenigen, über deren leiblich-seelische Unversehrtheit sie bestimmten, einzugehen oder sich bei den Untersuchungen der Ehekandidaten deren Vertrauen zu sichem. Dies muß jedoch als Akt politischer Opportunität aufgrund des wachsenden Widerstands gegen Zwangssterilisationen und 
Eheverbote verstanden werden. Die grundsätzliche, auf den professionell-wissenschaftlichen Status gegründete Hierarchie wurde dadurch nicht angetastet.

\section{Das öffentliche Gesundheitswesen im Nationalsozialismus als wissenschaftlich-technischer Apparat für die künstliche Selektion ${ }^{2}$}

Zur zentralen wissenschaftlich-technischen Bürokratie für die Geburten- und Rassenpolitik des NS-Staates wurde das öffentliche Gesundheitswesen umgebaut. Daß der Nationalsozialismus in dieser Weise seine geburten- und rassenpolitischen Ziele realisieren würde, war nicht von vornherein festgelegt oder geplant. Rassenhygiene und Antisemitismus waren zentrale Elemente der nationalsozialistischen Politik: programmatische Erklärungen hatten dies den Zeitgenossen deutlich gemacht. Wie jedoch die konkrete Durchsetzung dieser Politik aussehen würde, war in den ersten Monaten offen. Neben lokalen, in $\gg$ Selbsthilfe « beginnenden eugenischen und rassistischen Aktivitäten - teilweise angesiedelten bei städtischen Gesundheitsämtern existierten verschiedene Vorschläge darüber, wie die neue Politik reichsweit organisiert werden sollte.

Die Mehrzahl dieser Organisationspläne sah für die rassenhygienische und rassistische Politik Institutionen vor, die nicht mit der Gesundheitspflege gekoppelt waren. Wäre der Selektionsapparat in Richtung dieser Vorschläge organisiert worden, so ist anzunehmen, daß die vorhandenen Einrichtungen der kommunalen oder bezirklichen Gesundheitsfürsorge neben neuen staatlichen »Rassenämtern « weiter existiert hätten, möglicherweise auf dem niedrigen Finanzniveau der Wirtschaftskrise. Einige Beratungsstellen hätten vielieicht - außer den politisch und rassistisch motivierten Entlassungen der ärztlichen Leitung - wegen weiterer Mittelkürzungen schließen müssen. Aus der Kostenperspektive wie unter dem Blick winkel der im Zuge der Wirtschaftskrise zunehmend von Professionellen vertretenen Anschauung, daß etwa Tuberkulose-, Alkoholiker-, oder Blindenfürsorge mit »Minderwertigen « zu tun habe, für die zuviel ausgegeben werde, hätte die rassenhygienische Doktrin auch durch die Errichtung separater Erbpflege- oder Rassenämter umgesetzt werden können. Das aber geschah in dieser Form nicht. Stattdessen bestimmte das Gesetz über die Vereinheitlichung des Gesundheitswesens vom 3. Juli 1934 (GVG) und die dazu erlassenen drei Durchführungsverordnungen (DVO) mit Wirkung ab dem 1.5.1935 die Integration der »Bevölkerungs- und Rassenpolitik « in ein auszubauendes verstaatlichtes öffentliches Gesundheitswesen. ${ }^{3}$

Die Reform war in hohem Maße das Werk des Medizinalbeamten Arthur Gütt (18911949), durch dessen Berufung in das Reichsministerium des Innern (RMdI) die Entscheidung für den Ausbau und die Umstellung des öffentlichen Gesundheitswesens zum rassenhygienischen Selektionsapparat gefallen war. Gütt war lange Jahre (rassen- und bevölkerungs-) politisch engagiert in der deutschvölkischen Nationalen Freiheitsbewegung, berufspolitisch war er verankert im Verein preußischer Medizinalbeamter, der die Güttsche Konzeption einer staatlichen Bevölkerungspolitik 1932 zur Grundlage seiner gesundheitspolitischen Reformvorschläge machte (Gütt 1932). 
Durch das GVG wurde sukzessiv überall in Deutschland Realität, was im Rahmen der ärztlichen Untersuchungen für ein Ehestandsdarlehen zuvor unsystematisch und unzureichend begonnen hatte: das Sammeln und Auswerten aller Informationen aus jedem Zweig der Gesundheitsfürsorge für die eugenische und rassistische Selektion. ${ }^{4}$ $\mathrm{Daß}$ dies einer der Hauptgründe für die »Vereinheitlichung des Gesundheitswesens « war, belegen - neben der Arbeits- und Organisationsweise der Gesundheitsämter selbst, auf die noch einzugehen sein wird - Gütts hartnäckig vertretene Positionen in den gut einjährigen zähen Verhandlungen mit dem Finanzministerium, dem Deutschen Gemeindetag und Preußen bis zur Verabschiedung des Gesetzes, seine Äußerungen in berufsöffentlichen Versammlungen und Schriften bis hin zu seiner Argumentation in der Auseinandersetzung mit dem »Reichsärzteführer Wagner um die Übertragung der Gesundheitsfürsorge vom Staat auf die Partei, die sich in einem Schriftwechsel mit Himmler von 1938 befindet:

„Wenn im dritten Reich die Gesundheitspflege und die Gesundheitsfürsorge durch Reichsgesetz zur Pflichtaufgabe des öffentlichen Gesundheitsdienstes und damit zur Staatsaufgabe erklärt worden ist, so geschah dies vorwiegend aus dem Grunde, um die Ermittelungen und Erfassungen, die bei Abhaltung der ärztlichen Fürsorgesprechstunden gewonnen werden, auch bei der Durchführung der Aufgaben auf dem Gebiet des Gesundheitsschutzes wie der Erb- und Rassenpflege und Bevölkerungspolitik mit zu verwenden. $\ll^{5}$

Genau hier lag ein Stück Kontinuität mit dem ansonsten schärfstens bekämpften »Weimarer System «, dem der Nationalsozialismus die Anerkennung in einem Punkt nicht verweigerte: Es habe die »Ausdehnung der fürsorgerischen Tätigkeit auf den einzelnen Menschen und die Familie vorbereitet und ermöglicht« (Gütt 1938, S. 8). Um die Bedeutung des Apparates für die Durchführung der Politik zu ermessen, soll im folgenden seine Struktur in Grundzügen skizziert werden. Das GVG beinhaltete vertikale und horizontale »Vereinheitlichung der Gesundheitsverwaltung «.

Auf der unteren Verwaltungsebene wurde der staatliche Gesundheitsschutz (Medizinalpolizei) mit den jeweils vorhandenen kommunalen oder bezirklichen Zweigen der gesundheitlichen Vor- und Fürsorge sowie der neuen »Erb- und Rassenpflege« verknüpft. In Kreisen ohne Gesundheitsamt wurde ein solches eingerichtet. Bei den neuen Ämtern war aus Kostengründen ein sukzessiver Ausbau der einzelnen Fürsorgezweige vorgesehen. Vordringlich sollten diejenigen Bereiche geschaffen werden, die bisher noch nicht abgedeckt waren: Erb- und Rassenpflege also zunächst, außer in den Städten, deren Eheberatungsstellen nicht geschlossen worden waren. Großer Wert - besonders in ländlichen Regionen - wurde auch auf den Ausbau der ärztlichen Mütterberatung und Säuglingsfürsorge gelegt. Das Gesundheitsamt war nach dem Gesetz alleiniger Träger der ärztlichen Fürsorge- und Beratungsstellen in seinem Bezirk; bisherige Einrichtungen sollten übernommen werden. Wegen mangelnder Finanzkraft und Personalstärke konnte dies erst nach und nach geschehen, was mit der höheren Verwaltungsbehörde genau abzusprechen war. Spezialfürsorgeeinrichtungen Dritter konnten jedoch aus bestimmten Gründen bestehen bleiben, z.B. Beratungsstellen für Geschlechtskranke der Landesversicherungsanstalten oder Tuberkulosefürsorgestellen der Krankenkassen. Sie waren allerdings zur direkten Zusammenarbeit mit dem Gesundheitsamt verpflichtet (Meldungen oder den Austausch von 
Akten). Im Lauf der Jahre wurden die Daten dieser Fürsorgestellen und bestimmte Fürsorgezweige selbst dem Gesundheitsamt bzw. seiner Zentralkartei »einverleibt«. Ansonsten hatten sich die Kommunen « ... jeder eigenen Tätigkeit ... auf den ... den Gesundheitsämtern übertragenen Gebieten zu enthalten $\ll^{6}$, es sei denn, ein kommunales Gesundheitsamt war als solches vom RMdI anerkannt. Dann waren »die (ihm) obliegenden amtlichen Aufgaben als Auftragsangelegenheiten (zu) erledigen . $^{7}$ An $\mathrm{Zu}-$ sammenarbeit zwischen dem Gesundheitsamt und den örtlichen Machtträgern von Staat und Partei in Gemeinden, Kreis und Bezirk war vorgeschrieben: Kooperation mit dem Bürgemeister bzw. Landrat, dem Wohlfahrtsamt, der Gemeindeschwester, Jugendamt und Standesamt, der Polizei und den Gerichten sowie den »gesundheitlichen Einrichtungen der NSDAP $«$, hier insbesondere mit den IIilfsstellen für Mutter und Kind der nationalsozialistischen Volkswohlfahrt (NSV) und dem Amt für Volksgesundheit, der Gesundheitsorganisation der NSDAP (Reeg 1988).

Eheberatungsstellen - auch die weitergeführten aus den zwanziger Jahren - und örtlich gegründete Rassenämter aus der frühen Phase der nationalsozialistischen »Machtergreifung« wurden in der Beratungsstelle für Erb-und Rassenpflege beim Gesundheitsamt zusammengefaßt. In den 1935 und 1938 erlassenen Grundsätzen für die Tätigkeit der Beratungsstellen für Erb- und Rassenpflege sowie den Grundsätzen für die Beurteilung der Erbgesundheit von 1940 wurde ihre Arbeit festgelegt, ausgerichtet nach einheitlichen Prinzipien, ausgestattet mit einheitlichen Arbeitsmitteln für das gesamte Reichsgebiet. Unabhängige ärztliche (Ehe-)Beratungsstellen außerhalb der Gesundheitsämter waren verboten, nicht hingegen die Verbindung von »erbärztlicher « Praxis mit wissenschaftlicher Forschung, wie sie z.B. in der erbbiologischen Poliklinik im Kaiserin-Auguste-Viktoria-Haus in Berlin-Charlottenburg (KAVH) oder im Institut für Erbbiologie in Frankfurt am Main stattfand. Die im KAVH eingerichtete Poliklinik fungierte z.B. als »Beratungsstelle für Erb- und Rassenpflege « für zwei Bezirke des Gesundheitsamtes Berlin-Charlottenburg (Dubitscher 1940/41; Verschuer 1939). Damit war zugleich über die gesundheitsamtliche Tätigkeit hinaus der Zugang zu Menschen als Forschungsobjekten gesichert, derer die moderne genetische Anthropologie, die Erbpathologie wie die Eugenik dringend bedurfte: eine Referenz des Staates an die Wissenschaft, die im Gegenzug die Erbforschung vorantrieb, um den Kreis der Zwangszusterilisierenden ausweiten zu können.

Vertikal erfolgte die »Vereinheitlichung « durch die Zentralisierung der Gesundheitsverwaltung. In jedem Stadt- und Landkreis wurde bis zum 1.4.1935 ein (in der Regel) staatliches Gesundheitsamt eingerichtet. Es stand unter der Leitung eines (in der Regel) staatlichen Medizinalbeamten, der von nun an - übrigens bis heute - einheitlich $»$ Amtsarzt« hieß.

Auch die verbliebenen kommunalen Gesundheitsämter mit kommunalem ärztlichen Leiter sowie die kommunalen Gesundheitsämter, die von einem staatlichen Amtsarzt geleitet wurden, unterstanden den zentralen Weisungen des RMdI. Im Oktober 1938 gab es 650 staatliche Ämter, 51 kommunale Ämter mit staatlichem Amtsarzt als Leiter, 38 kommunale Ämter mit kommunalem Leiter, zusammen also 739 Gesundheitsämter »im Altreich« - das hieß zu diesem Zeitpunkt ohne Österreich. ${ }^{8}$ 
Schließlich war die Etablierung eines Gesundheitsamtes in jedem Kreis selbst ein Schritt zur Angleichung der unterschiedlich ausgeprägten Infrastrukturen des öffentlichen Gesundheitswesens einzelner Länder, Regionen und Kommunen, besonders der Differenzen zwischen Stadt und Land. Der nationalsozialistische Staat schuf die Voraussetzungen für die Medikalisierung »rückständiger«bzw. bisher von ärztlichen Fürsorgestellen freier geographischer wie lebensweltlicher Bereiche und stellt somit ein Stück Verallgemeinerung ärztlich-wissenschaftlicher Zuständigkeit für Sexualund Geburtenkontrolle sowie für die familiale Frauenarbeit dar, in Deutschland gekoppelt mit massiven Körpereingriffen.

Die Geschäftsführung jedes einzelnen Gesundheitsamtes unterlag reichsweit allgemeinen Vorschriften für den amtlichen Schriftverkehr, die Geschäftsbücher (Reisetagebuch, Terminkalender, Inventarverzeichnis, Aktenverzeichnis, Gebührenverzeichnis und bestimmte Übersichten), die Listenführung, die Registratur, bis hin zu Postsendungen. Es gab die gleichen Listen, Formblätter und formalisierte ärztliche Untersuchungsbögen für verschiedene Anlässe. Schließlich schrieben Erlasse zwingend und fakultativ vom Gesundheitsamt zu beziehende Bücher und Zeitschriften vor. Einheitliche Berichtspflichten mit bestimmten Fristen, die über die Mittelinstanz an das RMdI oder das Reichsgesundheitsamt weitergeleitet werden mußten, darunter auch Daten für die Medizinalstatistik und den Jahresgesundheitsbericht ${ }^{9}$, waren ein weiteres Element der Vereinheitlichung. 1938/39 existierten hier an die 50 Formbogen, allerdings noch keine allgemeinverbindlichen für jeden Bereich der Gesundheitsfürsorge. Ausgearbeitet hingegen war ein Formbogen für die amtsärztlichen »Monatsbericht(e) über die Durchführung des Gesetzes zur Verhütung erbkranken Nachwuchses, des Ehegesundheitsgesetzes und des $\S 6$ der 1. Verordnung zum Blutschutzgesetz« an den Regierungspräsidenten bzw. die Landesregierung, die von diesen binnen zehn Tagen an das RMdI weiterzuleiten waren. ${ }^{10}$ Hier waren Zahlenangaben zu machen über die von den »ermächtigten Krankenanstalten vorgenommenen Unfruchtbarmachungen auf Grund von Erbgesundheitsgerichtsbeschlüssen a) durch chirurgischen Eingriff«, getrennt nach Männern und Frauen, »b) durch Strahlenbehandlung « (unterteilt in: Röntgenbestrahlung oder Radiumbestrahlung), getrennt nach »Frauen « und »Frauen unter 38 Jahren «; über »Schwangerschaftsunterbrechungen aus erbpflegerischen Gründen «, über »bei der Durchführung der Unfruchtbarmachung und Schwangerschaftsunterbrechung eingetretenen Todesfälle«, an das Gesundheitsamt gemeldete »Schwangerschaftsunterbrechungen aus gesundheitlichen Gründen« sowie über angezeigte Fehl- und Frühgeburten; schließlich über Ehetauglichkeitsuntersuchungen und ihr Ergebnis: ausgestellte und verweigerte Ehetauglichkeitszeugnisse, unterteilt nach rassischen und eugenischen Ablehnungsgründen. Diese Zahlen erschienen nie im Jahresgesundheitsbericht, weil sie der Geheimhaltung unterlagen.

Außerdem existierten reichsweit gleiche Aufsichtsbestimmungen der Länder bzw. Provinzen über die Gesundheitsämter. 
Kreuzung als Grundmuster der Arbeitsmethoden und der Büroorganisation

»Erb- und Rassenpflege einschließlich Eheberatung « wurde zum einen als eigene Beratungsstelle etabliert, in der ein- oder zweimal wöchentlich Sprechstunden abzuhalten waren, zum anderen aber begründete sie den Blickwinkel, unter dem die gesamte »Arbeit am einzelnen Menschen« im Gesundheitsamt stehen sollte. Auf der Dienstversammlung der preußischen Medizinalräte und -dezernenten im Februar 1935, die ganz im Zeichen der kommenden rassistischen Reform des öffentlichen Gesundheitswesens stand, betonte Herbert Linden, daß es nicht möglich sei, die »rassenhygienische Betrachtungsweise « bei den »bisher als sozialhygienisch bezeichneten « Arbeiten des Gesundheitsamtes außer Acht zu lassen: "... die Schulgesundheitsfürsorge, die Mütter- und Kinderberatung (sic! GC.), die Fürsorge für Tuberkulöse, Geschlechtskranke, körperlich Behinderte, Sieche und Süchtige (müssen) in rassenhygienischem Geiste betrieben werden « (Linden 1935, S. 3). Er warnte vor der $\gg \mathrm{Ge}-$ fahr « - und sah hier häufige Verstöße - daß »besonders in spezialistisch betriebenen Fürsorgezweigen « der »rassenhygienische Geist « häufig »hinter Gesichtspunkte des Fachgebiets zurücktrete«. Neben dem »neuen Geist « in der Gesundheitsfürsorge legte Linden den versammelte Medizinalbeamten weitere »Beziehungen zwischen der Erb- und Rassenpflege und den sozialhygienischen Arbeitsgebieten « dar, »nämlich (die) Beibringung des für die Arbeit auf erbpflegerischem Gebiet unbedingt notwendigen Unterlagenmaterials. Jede Beratung in der Fürsorgesprechstunde und jeder Hausbesuch müssen dazu ausgenutzt werden, das für die Beurteilung des Erbwerts der einzelnen Familie notwendige Material zusammenzutragen« (Linden 1935, S.4). $\mathrm{Da}$ diese programmatischen Erklärungen - niedergelegt als Aufgaben des Gesundheitsamtes in den Durchführungsverordnungen - auch umgesetzt wurden, belegen Aussagen wie z.B. die folgende über Säuglingsfürsorge von 1938:

»Schließlich bietet die Säuglingsfürsorge, insbesondere der erste Hausbesuch der Gesundheitspflegerin, eine willkommene Gelegenheit, sich in zwangloser Weise von den erbbiologischen Verhältnissen der Familie ein Bild zu machen. Während der Besuch der Gesundheitspflegerin, wenn er lediglich zur Feststellung einer Erbkrankheit erfolgt, meist nicht gern gesehen ist und infolge ablehnenden Verhaltens der Befragten oft nicht zu dem gewïnschten Resultat führt, werden die Ermittlungen eher von Erfolg sein, wenn sie sich unauffällig an eine vorherige wirkliche Beratung der Mutter anschließen, die dieser auch etwas Positives gibt.« (Hoffmann, S. 4)

Ziel der Tätigkeit der«Beratungsstelle für Erb- und Rassenpflege« war die Ermittlung und Beurteilung des Erbwertes einer Person, um entsprechende Maßnahmen zu verfügen oder zu empfehlen. Sie unterschied sich von den anderen Zweigen der Gesundheitsfür- und Vorsorge dadurch, daß sie ihre Diagnosen über die Klientel durch den Zugriff auf möglichst alle Familienmitglieder gewann: »Im Gegensatz zu den bisherigen Beratungsstellen z.B. für Tuberkulöse, Geschlechtskranke usw. hat die Beratungsstelle für Erb- und Rassenpflege neben dem augenblicklichen Gesundheitszustand des zu Beratenden vor allem seine Erbbeschaffenheit zu erforschen «, heißt es in den vom RMdI erlassenen Grundsätzen. ${ }^{11}$ Dazu sei es »notwendig, sich zu unterrichten, ob in der Sippe des Betreffenden Erbkrankheiten ... oder besonders vortreff-

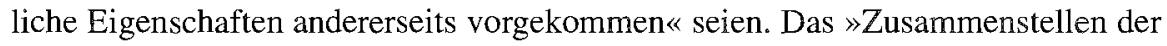


hierfür notwendigen Personalangaben, der erbbiologischen sowie sonstigen medizinischen Tatsachen « erfolge durch das Aufstellen einer Sippentafel. Zur Überprüfung der Angaben »des zu Beratenden « über seine Familie - ob nicht etwa ein krankes Familienmitglied verschwiegen wurde - sollten u.a. Auskünfte bei kommunalen und staatlichen Behörden wie bei der NSDAP eingeholt werden, außerdem bei Ärzten, Krankenhäusern, Anstalten und sonstigen Stellen. Damit wandten die Gesundheitsämter in der Beratungsstelle für Erb- und Rassenpflege das moderne vererbungstheoretische Paradigma praktisch an, bis hin zur Verwendung des wichtigsten Arbeitsmittels der naturwissenschaftlichen Familienforschung, der Sippentafel (vgl. z.B. Grotjahn 1926). Der in der Zeit des Nationalsozialismus inflationäre Gebrauch des Wortes »erbbiologisch « macht nur vor diesem Hintergrund Sinn - sofern man ihn überhaupt verstehen will und nicht als Nazi-Floskel behandelt: Die genetische Konstituierung des Individuums kann per se nicht isoliert erfolgen, sondern nur im Kontext der Erfassung und Begutachtung der physischen, psychischen und sozialen Existenz der Familienmitglieder, die über gemeinsame Erbanlagen und Merkmale durch »Fortpflanzung « verbunden sind. Die Genealogie des jeweiligen Familienverbandes geriet auf diese Weise erstmalig in den amtsärztlichen und damit staatlichen Gesichtskreis. In der auf Basis der »erbbiologischen Ermittlungen« erstellten Sippenregistratur fanden die internen Verwandtschafts- und Familienstrukturen ihr abstraktes Abbild. Auch die mit dem Personenstandsgesetz 1937 eingeführte FamilienbuchFührung anstelle der zuvor individuell geführten Geburten-, Heirats-und Sterberegister in den Standesämtern hängt damit engstens zusammen. Wenn Abstammung und Vererbung so wichtig wurden, mußten auch Daten jedes einzelnen Menschen im Familienzusammenhang registriert und abrufbar sein; außerdem hätten die Amtsärzte weit weniger Zugriffsmöglichkeiten gehabt, zumal im Lauf der Zeit die Klientel zu Recht immer mehr verschwieg, was ihr oder Familienangehörigen gefährlich werden könnte.

Erbbiologie als Methode bestimmte nicht nur die Arbeit und den Blick auf die Menschen im Gesundheitsamt, sondern war darüber hinaus Muster seiner bürokratischen Organisation. Alle »bei den Gesundheitsämtern vorhandenen Untersuchungsergebnisse und Vorgänge « waren in einer »erbbiologischen Kartei « zu sammeln. ${ }^{12}$ Wurde in der neueren Forschung über diese Erbbestandsaufnahme berichtet, so galt das Interesse zum einen ihrem Erfassungscharakter (Bock 1986; Roth 1984), zum anderen der durch sie vermittelten Instrumentalisierung der Gesellschaft zum Großlabor (Weingart/Kroll/Bayertz 1989). Hier soll das Augenmerk auf die immanente Rationalität ihrer Struktur und Funktion gelegt werden. Die Erbbestandsaufnahme war keine isolierte Praxis in einem Seitenraum des Amts, sondern »ärztliches Arbeitsmittel « und bürokratische Grundlage des Gesundheitsamtes. Sie war untrennbar mit der Abwicklung und Durchführbarkeit der Ehe- und Sexualkontrollen verknüpft. Es ist kein Zufall, daß im medizinisch-juristischen Gesetzeskommentar zum Blutschutzund Ehegesundheitsgesetz die grundlegenden Erlasse und Vorschriften über die $B e$ ratungsstellen für Erb- und Rassenpflege sowie das Rundschreiben des RMdI betr. Richtlinien für die ärztlichen Untersucher der Ehestandsdarlehensbewerber vom 16. 
März 1934 abgedruckt sind sowie ausführliche Erläuterungen für die Eheberatung gegeben werden.

Sukzessive sollten die Daten und Untersuchungsergebnisse aller Personen, die freiwillig oder unfreiwillig mit dem Gesundheitsamt zu tun hatten, auf zwei Karteikarten fixiert und diese mit der einzurichtenden Sippenakte registraturtechnisch verknüpft werden. Sie bildeten das organisatorische und funktionelle Rückgrat der alltäglichen Arbeit eines jeden Amtes. Die Registratur als »ärztliches Arbeitsmittel « war analog der Wissenschaft strukturiert; Aufgabe der Gesundheitsämter im Nationalsozialismus war die praktisch-politische Umsetzung.

\section{Aufbau und Inhalt der erbbiologischen Organisationsstruktur}

Grundbausteine waren eine Karteikarte in zweifacher Ausfertigung pro Person und die Sippenregistratur pro Familie, die durch Zahlenverweise miteinander verbunden waren. Die erste Ausfertigung der Karteikarte war zunächst Suchkarte für alle Personen, $\gg$ mit denen sich das Gesundheitsamt eingehender befaßt hat «, diente der Übersicht über die dabei angelegte Akte und war »Übersichtsplan für alle Maßnahmen, deren wichtigste durch Bereiterung der entsprechenden Felder des oberen Randes der Karteikarte fortlaufend verfolgt werden können (z.B. die Aussetzungen der gerichtlich angeordneten Unfruchtbarmachungen). ${ }^{13}$ Die Karte war zunächst für alle Personen anzulegen, die in der Beratungsstelle für Erb-und Rassenpflege untersucht und über die ermittelt wurde. Ab 1938/39 sollte sie als Wohnortkartei zur Zentralkartei eines jeden Amtes ausgebaut werden. Die Zweitschrift der Karte war per Post an das Gesundheitsamt des Geburtsorts zu schicken oder bei gleichem Wohn- und Geburtsort als getrennte Geburtsortkartei einzurichten. Diese bildete »Beginn und Grundlage der erbbiologischen Bestandsaufnahme der Gesamtbevölkerung «. Sie hatte den Zweck der »Auskunfterteilung bei Eheberatungen, Anträgen auf Unfruchtbarmachungen usw. « ${ }^{14}$ Für im Ausland Geborene war das Reichsgesundheitsamt die zentrale Sammelstelle der Zweitschrift. Ab 1938 wurden in Berlin, Hamburg, Sachsen, Thüringen und dem Saarland zentrale Geburtsortkarteien geführt, später wurden bis fast zum Kriegsende weitere zentrale Geburtsortkarteien in folgenden annektierten Ländern eingerichtet: in der Tschechoslowakei für den »Reichsgau Sudentenland« in Reichenberg, die »`Zentralstelle für die Erbkartei der deutschen Bevölkerung in Böhmen und Mähren< in Prag. Ab Anfang 1943 waren in Elsaß, Lothringen und Luxemburg Gesundheitsämter errichtet, die die Geburtsortkarteien von dort jeweils geborenen Personen führten, ebenso in Kärnten und in der Steiermark. Die Zentrale Geburtsortkartei für den besetzten und nicht dem »großdeutschen Reich« zugeschlagenen Teil Polens ( $\gg$ Generalgouvernement $\ll$ ) war in Krakau. Da mit Kriegsbeginn die Sterilisationsverfahren bis auf »eindeutige Fälle« eingestellt wurden, lag der praktische Zweck vornehmlich in der Absicht, die Eheschließungen zu überwachen. Vereinfachung und bessere Objektivierung waren die beiden kennzeichnenden Prinzipien einer Bürorationalisierung im Jahre 1938, die auf den ersten gewonnenen Erfahrungen beruhte. Die Karteikarte für Wohnort- und Geburtsortkartei wurden von 
der vorherigen Kurzdiagnostik über den auf ihr festgehaltenen Menschen zum funktionellen Nachweismittel für das schnelle Auffinden von Unterlagen über die Person; nur noch wenige, aber für die Selekteure grundlegende Klassifizierungen und Maßnahmen wurden auf der Karte verzeichnet. Ebenso dünnte man die Rubriken der Sippentafel aus und paßte ihren Aufbau dem Personenstandsgesetz vom 3.11.1937 an. ${ }^{15}$ Von den sehr ausführlichen Einzelvorschriften seien hier nur einige genannt, um deutlich zu machen, wie Erfassung und Selektion nach medizinisch-psychiatrischen, sexuellen, rassischen und sozialen Gesichtspunkten bereits im Bürosystem sich niederschlugen. In der Sippentafel waren auf einem Übersichtsblatt die Personenzeichen zu markieren mit jeweils bestimmten Farbstiften für Schwachsinn, Geisteskrankheit, Fallsucht, erbliche Taubheit, Alkoholismus, Selbstmord und körperliche Erbkrankheiten: die Diagnostik für Zwangssterilisation und eugenische Eheverbote. Ebenso war nach »Rasse « zu klassifizieren. Aufgrund der durch das Reichsbürger- und Blutschutzgesetz amtlich festgelegten Rassendefinitionen sollten ab 1938 auf der Karteikarte folgende Bezeichnungen verwendet werden:

») Jude< (im Sinne des $§ 2$ der Ersten Verordnung zum Reichsbürgergsetz vom 14. November 1935, RGB1 I, S. 1333, also auch Dreivierteljuden),

>jüd. M I (= jüdischer Mischling mit 2 volljüdischen Großeltern),

>jüd. M II (= jüdischer Mischling mit 1 jüdischen Großeltemteil).« Weiter lauteten die Anweisungen: $\gg$ Für nichtjüdische Fremdrassige gelten folgende Bezeichnungen:

$\mathrm{Zi}$ (= Zigeuner), Ne (= Neger), Mo (= Mongole), bei Mischlingen mit dem entsprechenden Zusatz >M I bzw, >M II .

Alle sonstigen Personen, die als nicht deutschen oder artverwandten Blutes anzusehen sind, sind in dieser Spalte als $>\mathrm{AF}<$ (= artfremd) oder $>\mathrm{AFM}<$ (= artfremder Mischling) zu bezeichnen« (Gütt 1939, S. 586)。

In der Spalte »sozial « sollten »bestimmte, für die Beurteilung der Gesamtpersönlichkeit entscheidende Bezeichnungen « eingetragen werden, »welche eine Zuordnung der betr. Person zu bestimmten, sozial auffälligen Gruppen erlauben «. Kürzel für folgende Klassifizierungen waren hier vorgeschrieben: kriminell, rückfällig kriminell (ohne Bagatellstrafen), schwer kriminell, Zuchthaus oder Todesstrafe, Fürsorgeerziehung, häufig wechselnder Geschlechtsverkehr, Prostituierte (wenn als solche gemeldet), wegen Geistesschwäche, Geisteskrankheit, Trunksucht, Verschwendung entmündigt, arbeitsscheu, sonst asozial (Gütt 1939, S. 593). Bemerkenswert ist schließlich: Alle Frauen und Mädchen, ob ledig, verheiratet, geschieden oder verwitwet, sollten mit ihrem Geburtsnamen geführt werden, organisationspraktischer Ausdruck ihrer wissenschaftlich fundierten »Gleichberechtigung «, der ihrem »gleichen « Anteil an »Fortpflanzung und Vererbung « durch die auch in der weiblichen Keimzelle liegenden Gene Rechnung trägt. Deshalb ist die »Sippe« der Frau genauso wichtig wie die des Mannes und bedarf eindeutiger Bestimmung, im Unterschied zu den patriarchal ausgerichteten (vorgenetischen) Familien-Stammbäumen, in denen die Ehefrauen jeweils allein in der männlichen Linie des Ehemannes aufgezeichnet erscheinen. Als »wichtigstes Anschlußmittel« waren die Systeme der Gesundheitsämter durch das Verschicken der Karteikarten für die Geburtsortskarteien untereinander zu vernetzen. Dies war wegen der wachsenden Binnenwanderung immer notwendiger geworden. Die Ämter kamen mit ihrem Erfassungssystem der zunehmenden Mobilität 
kaum nach: Rund ein Viertel der Bevölkerung zog mit beginnender Rüstungskonjunktur im Durchschnitt alle vier Jahre um. Die Freizügigkeit - besonders die »des Großstädters « - störe die Erbbestandsaufnahme empfindlich, hieß es 1940 (Drope und Vellguth) ${ }^{16}$ Ein Datenabtausch mit den Einwohnermeldeämtern wäre viel zu aufwendig gewesen. Außerdem waren für ein Gesundheitsamt mit einem Einzugsgebiet von z.B. 100000 Einwohnern nicht alle 25000 umgezogenen, sondern jene Personen und ihre Familienmitglieder primär von »Interesse «, die für Zwangssterilisationen und Eheverbote »vorgemerkt« oder die sonst als »auffällig« in der Rubrik »sozial《 erfaßt worden waren. »Daß wir uns ... zunächst und vordringlich mit der negativen Auslese befassen «, so Vellguth weiter, habe den »rein äußeren Grund, daß sich ein größerer Teil unserer erb- und rassenpflegerischen Maßnahmen heute auf diesen Personenkreis bezieht und daß der größte Teil der fördernden Maßnahmen zur Voraussetzung hat, daß die Betroffenen nicht zur negativen Auslese gehören. Grundsätzlich erstreckt sich unsere Arbeit auf die Gesamtbevölkerung « (Vellguth 1938, S. 500 f.) Die Erbbestandsaufnahme läßt sich in der Tat als eine »Gemeinschaftsarbeit allergrößten Stils, an dem jedes der 800 Gesundheitsämter in gleicher Weise beteiligt ist«, bezeichnen (Vellguth 1938, S. 497). Der lokale und überregionale Registratur-Verbund von Sippenakten, Wohnort- und Geburtsortkarteien wurde zur funktionellen Schnittstelle zwischen gesetzlichen Maßnahmen der »Auslese « und »Ausmerze « und dem Apparat: zwischen den Indikationen für Zwangssterilisation und Eheverbote, den vorgegebenen Kriterien für die Ablehnung oder Gewährung von Ehestandsdarlehen, Kinder- und Ausbildungsbeihilfen, sowie dem »Ehrenkreuz der deutschen Mutter « einerseits und den gesundheitsamtlichen Fürsorgezweigen, aus deren Klientel sich ein Großteil der »Objekte « für die entsprechenden Maßnahmen rekrutierte, andererseits. Was für die Klientel mündliche oder schriftliche Ausfragerei und die Erhebung von Befunden am eigenen Leib und ihrer sozialen Lebenswelt war, war gleichsam die »Eingabe« für den Apparat mit Auswirkungen auf den Betriebslauf in naher und ferner Zukunft. Ziel war, ein »Nachschlagewerk der negativen Auslese « zu schaffen, auf dessen Basis amtsärztliches Handeln (termingerecht) einzuleiten und Selektionsentscheidungen zu begründen waren. »Für die Zukunft muß die Erbbestandsaufnahme das wichtigste Material für die Erb- und Rassenpflege werden«, hieß es 1938. »Heute « sei die Anlage von Karteikarten und Sippentafeln noch vielfach die Folge der praktischen Maßnahmen, künftig jedoch sollten sich die praktischen Maßnahmen »aus dem bereits vorliegenden Material « ergeben. »Nicht weil wir einen Antrag auf Unfruchtbarmachung stellen, stellen wir eine Sippentafel auf, sondern wir stellen einen Antrag, weil sich die Notwendigkeit dazu aus unserem Material ergibt « (Vellguth 1938, S. 501).

Wie weit dies von seiner Anlage her höchst effektiv erscheinende Erfassungs- und Selektionssystem letztlich funktionierte, war abhängig vom jeweiligen Personalstand eines Gesundheitsamtes, den Amtsärzten, den Hilfsärzten und den Fürsorgerinnen. Zudem trugen die Ansprüche der lokalen wie zentralen Wohlfahrts-, Gesundheitsund Ärzteorganisationen der NSDAP und der Kommunen dazu bei, daß der Ausbau der Registratur unterschiedlich weit gedieh. Neben dem enormen Arbeitsaufwand 
- in Hamburg wurden z.B. eigens »Karteiführer« eingestellt - ist schließlich die Empfindlichkeit des Apparts selbst zu bedenken: ein gigantisches Werk und äußerst störanfällig, sei es nur ein Stocken im Ausfüllen der Karteikarten durch Personalmangel oder eine kleine Nachlässigkeit der Schreibkraft. Nicht zuletzt streuten die Betroffenen selbst aktiv und passiv, bewußt oder unbewußt Sand ins Getriebe: Die zunehmende Freizügigkeit, sei es im Zusammenhang mit dem wachsenden Arbeitskräftemangel im Zuge der Rüstungskonjunktur und dem Abwerben durch unternehmerische Lohnpolitik oder durch Untertauchen, schließlich der Krieg brachte den »Stand « des Erfassungssystems immer wieder neu ins Hintertreffen. Heute im Zeitalter von Computern und Telefaxe, sähe es allerdings anders aus. 1942 waren offensichtlich um die 10 Millionen Karteikarten erstellt (Bock 1986, S. 191 f.). Welche Personenkreise und Familien »verkartet« waren, ist unbekannt. Immerhin entsprach die Zahl der in den zwanziger Jahren z.B. von Fritz Lenz aufgrund der Medizinalstatistik geschätzten »negativen Auslese« der Reichsbevölkerung. Andererseits wurden etwa vier Millionen Männer und Frauen für ein Ehestandsdarlehen ärztlich untersucht und hätten entsprechend der Vorschriften ebenfalls »verkartet《 sein müssen. Die Ablehnungsquote war hier mit unter fünf Prozent gering.

Auch wenn das Ziel der Erbbestandsaufnahme der Gesamtbevölkerung nicht erreicht werden konnte, funktionierten die Gesundheitsämter angesichts der Millionen Untersuchungen, die für viele Menschen katastrophale Folgen hatten, dennoch auf schlimme Weise effektiv genug. In den letzten Kriegsjahren wurden Selektion und Mord dort praktiziert, wo Erfassung und Segregation längst stattgefunden hatten: in den russischen Kriegsgefangenenlagern, den Lagern für »slawische « Zwangsarbeiter und Zwangsarbeiterinnen, in den Säuglingsbaracken, in den Konzentrationslagern, in den Vernichtungslagern, in den »Heil- und Pflegeanstalten «. Die ab 1941 von allen Brautleuten geforderte Eheunbedenklichkeitsbescheinigung jedoch zeigt, daß die Politik der »Ausmerze« und der«Auslese « nicht von den Gesundheitsämtern in die Lager verschoben wurde, noch, daß sie für das öffentliche Gesundheitswesen zu Ende war: Herbert Linden - während derselben Zeit beschäftigt mit der Organisation der Anstaltsmorde (Aly 1985, S. 17) und eingeweiht in Fleckfieberversuche an KZ-Häftlingen (Mitscherlich, Mielke, S. 91 f.) - lobte diese Bescheinigungen als »erheblichen Fortschritt in der Erbpflege « im Hinblick auf die nach dem Ehegesundheitsgesetz vor jeder Eheschließung beizubringenden Ehetauglichkeitszeugnisse. Diese sollten nach dem Krieg von jedem Brautpaar im Standesamt vorgelegt werden (Linden 1942). ${ }^{17}$ Dazu ist es nicht mehr gekommen. Die 1946 in Württemberg eingeführte eugenische Eheberatung vor der Eheschließung durch Ärzte wurde wenig später aufgrund des geringen Erfolges bei den Brautpaaren wieder abgeschafft (Müller-Freienfels 1962). Das vom nationalsozialistischen Staat geschaffene öffentliche Gesundheitswesen hingegen hat - reföderalisiert und im Gesetz um »Rasse « und »NSDAP«, nicht aber um »Erb- ...pflege« bereinigt - Bestand bis heute. Die Erbkartei blieb, von »überflüssigem Beiwerk befreit «, zentrale Suchkartei (Kuhn 1966). 


\section{Die Bedeutung von Kreuzung in der Selektionspraxis oder: Die Taxierung des Paares}

Schließlich bestimmte das Kreuzungsexperiment das Verfahren der ärztlichen Selektion für Ehestandsdarlehen und Eheverbote. Nach dem modernen Vererbungsparadigma war es gar nicht anders möglich: Eheeignung wie die Ehetauglichkeit als eugenische Qualifikation konnten weder abstrakt noch »individualistisch « bestimmt werden, sondern verlangten neben der körperlichen Kontrolle der »Ehebewerber « jeweils die Ausleuchtung ihrer Familien. Analog der genetischen Theorie blickte der Amtsarzt auf das Paar als auf zwei Exemplare unterschiedlicher Erblinien in $\operatorname{der} F_{2}$ Generation und wandte in prognostischer Absicht virtuell das die moderne Genetik konstituierende Experiment, die Kreuzung, an. Aus diesem Grund kam es bei der Beurteilung entscheidend auf die Partner-Kombination an: ausschlaggebend war $\gg$ das Erbbild des anderen Partners sowie das Sippenbild beider Partner « (Gütt/Linden/ Maßfeller 1936, S. 66).

Ebenso wie das Klassifizieren und Kombinieren bei der Beurteilung der Ehevorhaben von »Mischlingen « verschiedenen »Grades« miteinander oder mit jüdischen oder nichtjüdischen Nicht-»Mischlingen « für rassistische Eheverbote oder Genehmigungen nach dem Blutschutzgesetz eine zentrale Rolle spielte ${ }^{18}$ - die »Grade «bildeten ja nichts anderes als das Generationen-Rassenmischungs-Verhältnis ab --, beruhten auch die eugenischen Eheverbote oder Genehmigungen nach dem Ehegesundheitsgesetz auf Klassifizierung und Beurteilung der durch das jeweils bestimmte Paar verkörperten »Kombinationen«:

1. ein Partner ist krank, der andere gesund;

2. ein Partner ist erblich belastet, der andere gesund;

3. beide Partner sind krank;

4. ein Partner ist krank, der andere erblich belastet;

5. beide Partner sind erblich belastet.«

(Gütt/Linden/Maßfeller 1936, S.134)

Die Kombinatorik ist bis heute eine der grundlegenden Methoden der humangenetischen Beratung. Der Unterschied zum Nationalsozialismus liegt weniger in der inzwischen fortgeschrittenen Kenntnis über einige körperliche Erbkrankheiten, sondern in den Rechtsfolgen der humangenetischen Beratung. Damit sind bei gleichbleibender wissenschaftlicher Grundlage die Probleme verlagert.

\section{Vergangenheit und Gegenwart: Ausblick}

Genetik und Humangenetik haben ihren sozialpolitischen Anspruch nicht aufgegeben, soweit es um die Verbesserung des gen pools geht. Die negative Eugenik soll dabei versuchen, »die Häufigkeit nachteiliger Gene zu verhindern«, die positive Eugenik, »die Häufigkeit vorteilhafter Gene zu erhöhen«. Nicht zuletzt aufgrund der Erfahrungen im nationalsozialistischen Deutschland wurde und wird von der internationalen Genetikergemeinde die Anwendung von Zwangseingriffen verurteilt. Sie 
setzt statt dessen darauf, »auf sozialer Ebene zu veranlassen, daß sich Genotypen, die ganz offensichtlich nachteilig sind, sich nicht fortpflanzen « (Strickberger 1988, S. 189). Da zudem präzisere Berechnungsgrundlagen ergaben, daß sich eine »Verbesserung « des gen pools durch Verminderung rezessiver pathogener Erbanlage qua »negativer Eugenik « innerhalb überschaubarer zeitlicher Dimensionen kaum herbeiführen läßt (van den Daele 1985), hat sich der sozialpolitische Anspruch auf die "positive« Eugenik als »Anreicherung « des gen pools verlegt. Nach wie vor ist die »Methode der Selektion von größter Bedeutung «. Weil »eugenische Maßnahmen, die diktieren, wer wen heiraten soll «, z.B. »gezielte Paarung « als untragbar abgelehnt wird, ist unter anderem ${ }^{19}$ Samenwahl statt Gattenwahl angesagt: »Nach dieser Methode würden weibliche Freiwillige sich entscheiden, sich durch Männer künstlich befruchten zu lassen, die schon lange tot sind, aber in hohem Maße über wünschenswerte Eigenschaften verfügten « (Strickberger 1988, S. 189). In der Vorstellung, daß man Paare beeinflussen solle, »für ihre Kinder eine sehr hochwertige genetische Ausstattung zu wünschen «-so ein anderer Vorschlag - treffen sozialpolitisches Engagement derGenetik, humangenetische Beratung und pränatale Diagnostik zusammen.

Im Gegensatz zur nationalsozialistischen Erb- und Rassenpflege ist eine humangenetische Beratung heute nicht gesetzlich zwingend vorgeschrieben; es existieren weder eugenische Eheverbote, noch können Sterilisation oder Abtreibung aus eugenischen Gründen auf legale Weise erzwungen werden. Die eugenische Indikation für einen Schwangerschaftsabbruch blieb jedoch seit der NS-Zeit erhalten und gewinnt angesichts fortgeschrittener pränataler Diagnosetechniken an Bedeutung. Schwangere Frauen werden inzwischen einem großen Entscheidungsdruck ausgesetzt; schon der Anwendung der Untersuchungsverfahren können sie sich nur noch schwer entziehen. Genetik, Gynäkologie und Perinatale Medizin rücken immer enger zusammen und treffen sich in dem Bemühen um das »gesunde Kind «. Es wird zum erwünschten Produkt, auf das die Gesellschaft und die Eltern Anspruch haben. Dahinter verbirgt sich letztendlich eugenisches Denken über »lebensunwertes Leben«.

Eine der wichtigsten Fragen, die schon fast überholt zu sein scheint, ist die nach dem Verhältnis von Medizin, Recht und Körper oder: Wie verhält sich die Freiheit der Human- und Biowissenschaften zur Freiheit der Person, dem wesentlichen Grundrecht in einer bürgerlich-demokratisch verfaßten Gesellschaft? Die Entwicklung geht offensichtlich dahin, daß die Freiheit der Person sich darin erschöpft, sich dem Denken und jeweiligen Kenntnis- und Technikstand der Wissenschaft freiwillig und »aufgeklärt« anzupassen. Eine menschenfeindliche Entwicklung.

\section{Anmerkungen}

1 Wenn Erwin Baur (1930) schreibt, »Die heutigen Menschenrassen sind alle untereinander kreuzbar und geben völlig fertile Bastarde«, verrät sich in der Tat das Denken des Experimentators - vollends dann, wenn er bedauert, daß $»$ Kreuzungsversuche zwischen Menschen und dem nächst verwandten Menschenaffen (Schimpanse etwa), mit Hilfe von künstlicher Befruchtung technisch ohne Schwierigkeiten ausgeführt werden könnten, ... bisher - leider - noch nicht ausgeführt« wurden, denn so kön- 
ne geklärt werden, ob »diese Kreuzungen zu lebensfähigen $F_{1}$-Individuen führen würden«, was er zwar für »wenig wahrscheinlich, aber durchaus nicht von vornherein für unmöglich « hielt. Zu Baur vgl. Gilsenbach 1990.

2 »Künstliche Selektion« im Gegensatz zu »natürlicher Selektion« und »künstlich« im Sinn von »ärztlicher Kunst«.

3 Gesetz über die Vereinheitlichung des Gesundheitswesens vom 3. Juli 1934, Reichsgesetzblatt (RGB1), S. 531; 1. DVO vom 6.2.1935, RGB1 I, S. 177; 2. DVO (Dienstordnung - Allgemeiner Teil) vom 22.2.1935, ebda., S. 215; 3. DVO (Dienstordnung - Besonderer Teil) vom 30.3.1935, ReichsMinisterialblatt für die Innere Verwaltung, S. 327 (Beilage zu Nr. 14). Zu den Phasen der Durchsetzung des GVG im einzelnen vgl. Labisch/Tennstedt 1984, S. 257-313.

4 Wie notwendig ein personell und finanziell gut ausgestatteter Apparat für die Durchführung rassenhygienischer Politik ist, zeige ich in meiner Dissertation (Czarnowski 1989) anhand der Probleme, die bei den überstürzt eingeführten ärztlichen Eheeignungszeugnissen für Ehestandsdarlehensbewerber/innen vor der Reform des öffentlichen Gesundheitswesens entstanden - Probleme, die weniger die Untersuchten als die Untersucher hatten, weil sie zu Recht fürchteten, daß ihnen viele Angaben verheimlicht wurden.

5 Abschrift eines undatierten 10seitigen dienstlichen Schreibens von Gütt als Leiter der Abteilung IV »an Herrn Minister durch die Hand des Herrn Staatssekretärs«, als Abschrift beigefügt dem vertraulichen Schreiben Gütt an Himmler vom 14.1.1938, Institut für Zeitgeschichte, MA 3 (1), Himmler Files Nr. 1302, Drawer 1, Folder 5, RFSS/Pers. Stab.

6 1. DVO \& 9, Gütt 1939, S. 144.

7 1. DVO $\$ 20-21$, Gütt 1939, S. 46.

8 Verzeichnis der Gesundheitsämter nach dem Stande vom 1. Oktober 1938, Gütt 1939, S. 329-357.

9 Der Jahresgesundheitsbericht wurde übrigens seit 1935 auf jeweils für alle Länder einheitlichen Vordrucken erstattet; damit wurde auch hier ein teilweise lange dauernder Partikularismus angeglichen: In Sachsen x.B. wurden seit 1867, in Preußen seit 1901 Jahresgesundheitsberichte erstellt (Gütt 1939, S. 88).

10 Abdruck eines ausgefüllten Monatsberichts einer mittleren Verwaltungsbehörde (Oldenburg) in Roth 1984, S. 77

11 Grundsätze für die Errichtung und Tätigkeit der Beratungsstellen für Erb- und Rassenpflege, Anlage 1) zum Runderlaß RuPrMdI vom 21.5.1935 - IV f 3060/1075 b -betrifft: Beratungsstellen für Erb-und Rassenpflege, in: Gütt/Linden/Maßfeller (1936) S. 286

12 \$33. DVO zum GVG (Dienstordnung für die Gesundheitsämter-Besonderer Teil) vom 30.3. 1935, Gütt 1939, S. 261; vgl. Bock 1986, S. 188-192; Roth 1984, S. 75 f.

13 Erläuterung zur Karteikarte, Anlage 5) zum RdErl RuPrMdI vom 21.5.1935, Gütt/Linden/Maßfeller (1936) S. 296.

14 Erläuterung zur Karteikarte, Gütt/Linden/Maßfeller (1936) S. 296.

15 Grundsätze für die Tätigkeit der Beratungsstellen für Erb- und Rassenpflege in den Gesundheitsämtem, gedruckt als Anlage zum RdErl RuPMdI vom 1.4.1938 - IV b 1289/38/1075 b, S. 109, in: Gütt 1939, S. 555 f., S. 575-607.

16 Auf der anderen Seite nutzten sie die in »jeder zivilisierten Großstadt« viel mehr als auf dem Lande vorhandenen »Einrichtungen, bei denen Menschen, die zur negativen Auslese gehören, erfaßt werden « als Informationsquellen zur Gruppenerfassung aus.

17 Das Versagen eines Ehetauglichkeitszeugnisses zog entsprechend \& 2 Ehegesundheitsgesetz ein Eheverbot nach sich. Dieser Paragraph war 1936 noch nicht generell in Kraft gesetzt worden, sondern wurde selektiv gehandhabt in Abhängigkeit vom Blick des Standesbeamten und dem Stand der erbbiologischen Erfassung im Gesundheitsamt, denn jedes Aufgebot wurde an das Gesundheitsamt weitergeleitet.

18 1. AVO BhutschG v. 14.1 1.1935, Erläuterungen in Gütt/Linden/Maßfeller (1936) S. 219-225.

19 Samenbanken, Klone, Parthenogenese oder Genchirurgie gehören zum Arsenal positiver Eugenik. 


\section{Literatur}

Aly, G. (1985): Der saubere und der schmutzige Fortschritt, in:Reform und Gewissen. Euthanasie im Dienst des Fortschritts (Beiträge zur nationalsozialistischen Gesundheits-. und Sozialpolitik Bd. 2) S. 9-78

Baur, E. (1930): Einführung in die Vererbungslehre, 7. 11. völlig neu bearb. Aufl., Berlin

Baur, E., Fischer, E., Lenz, F. (1936): Menschliche Erblehre und Rassenhygiene. Bd. 1: Menschliche Erblehre, 4. neubearb. Aufl., München

Bergmann, A. (1988): Die »Rationalisierung der Fortpflanzung «: Der Rückgang der Geburten und der Aufstieg der Rassenhygiene/Eugenik im deutschen Kaiserreich 1871-1914, Phil. Diss. Freie Universität Berlin

Bergmann, A., Czarnowski, G., Ehmann, A. (1989): Menschen als Objekte humangenetischer Forschung und Politik im 20. Jahrhundert, in: Ärztekammer Berlin in Zusammenarbeit mit der Bundesärztekammer (Hg.), Redaktion: Pross, C., Aly, G., Der Wert des Menschen. Medizin in Deutschland 1918- 1945, (Reihe deutsche Vergangenheit 34), S. 121-142

Bock, G. (1986): Zwangssterilisation im Nationalsozialismus. Studien zur Rassenpolitik und Frauenpolitik (Schriften des Zentralinstituts für sozialwissenschaftliche Forschung der Freien Universität Berlin, Bd. 48), Opladen

Corea, G. (1986): Muttermaschine. Reproduktionstechnologien - Von der künstlichen Befruchtung zur künstlichen Gebärmutter, Berlin

Czarnowski, G. (1989): Ehe- und Sexualpolitik im Nationalsozialismus: Medizin und Politik in ihrer Bedeutung für das Geschlechterverhältnis, Phil. Diss. Freie Universität Berlin

Daele, W. van den (1985): Mensch nach Maß? Ethische Probleme der Genmanipulation und Gentherapie, München

Drope, D., Vellguth, H. (1940): Erbbestandsaufnahme in der Großstadt, in: Der öffentliche Gesundheitsdienst, Ausg. B, 6. Jg., S. 261 f.

Dubitscher, F. (1940/41): Praktische Erb- und Rassenpflege, Erfahrungen aus der Poliklinik für Erb- und Rassenpflege Berlin, in: Der öfentliche Gesundheitsdienst, Ausg. B, 6. Jg., S. 545-569

Entres, L. (1928): Die Ursachen der Geisteskrankheiten. Vererbung, Keimschädigung, in: Bumke, $O$. (Hg.), Handbuch der Geisteskrankheiten, 1. Bd. Allgemeiner Teil I

Eugenics in Britain (1979): Annals of Science, Special Issue, Vol. 36

Faith Weiss, S. (1987): The Race Hygiene Movement in Germany, in: Osiris. A Research Journal devoted to the History of Science and its Cultural Influences, 2nd series, S. 193-236

Fischer, E. (1936): Schlußansprache, in: Harmsen, H., Lohse, F. (Hg.), Bevölkerungsfragen. Bericht des Internationalen Kongresses für Bevölkerungswissenschaft Berlin 26. August - 1. September 1935, München (Reprint Neudeln-Liechtenstein 1969)

Gilsenbach, R. (1990): Erwin Baur, Eine deutsche Chronik, in: Arbeitsmarkt und Sondererlaß. Menschenverwertung, Rassenpolitik, Arbeitsamt (Beiträge zur nationalsozialistischen Gesundheits- und Sozialpolitik Bd. 8) S. 184-197

Gordon, L. (1977): Women's Body, Women's Right. A Social History of Birth Control in America, New York

Graham, L. (1977): Science and Values: The Eugenics Movement in Germany and Russia in the 1920s, in: The American Historical Review, Vol. 82, S. 1133-1164

Grossmann, A. (1984): The New Woman, the New Family, and the Rationalization of Sexuality: The Sex Reform Movement in Germany 1928 to 1933, Ph. D. Rutgers University, New Jersey

Grossmann, A. (1987): Berliner Ärztinnen und Volksgesundheit in der Weimarer Republik: Zwischen Sexualreform und Eugenik, in: Eifert, C., Rouette, S. (Hg.), Unter allen Umständen. Frauengeschichte(n) in Berlin, Berlin, S. 183-217

Grotjahn, A. (1926): Die Hygiene der menschlichen Fortpflanzung. Versuch einer praktischen Eugenik, Berlin und Wien

Gütt, A. (1932): Bevölkerungspolitik und öffentliches Gesundheitswesen, 19. Hauptversammlung des deutschen Medizinalbeamtenvereins in Eisenach, 22. und 23.9.1932, in: Zeitschrift für Medizinalbeamte, Jg. 45. S. 451-472

Gütt, A. (1938): Der Aufbau des Gesundheitswesens im Dritten Reich (Schriften der Deutschen Hochschule für Politik 10/11), 4. überarb. Aufl. 
Gütt, A. (1939):Der öffentliche Gesundheitsdienst. Erläuterungen zum Gesetz über die Vereinheitlichung des Gesundheitswesens vom 3. Juli 1934 nebst Durchführungsverordnungen, Gebührenordnung und Anhang mit Erlassen (Handbücherei für den öffentlichen Gesundheitsdienst Bd. 1), völlig neu bearb. Aufl., Berlin

Gütt, A., Linden, H., Maßfeller, F. (1936): Blutschutz- und Ehegesundheitsgesetz. Gesetz zum Schutze des deutschen Blutes und der deutschen Ehre und Gesetz zum Schutze der Erbgesundheit des deutschen Volkes nebst Durchführungsverordnungen sowie einschlägigen Bestimmungen. Dargestellt, medizinisch und juristisch erläutert von ..., München

Hoffmann, Dr. (1938/39): Organisation der Säuglingsfürsorge in einem ländlichen Gesundheitsamt, in: Der öffentliche Gesundheitsdienst, Ausg. B, 4. Jg., S. 1-11

Kuhn, R. (1966): Geschäftsführung, in: Daniels, Josef u.a. (Hg.) Das öffentliche Gesundheitswesen, Bd. I: Gesundheitsverwaltung, hg. von F. Pürckhauer und J. Stralau, Teil A: Grundlagen, Stuttgart

Labisch, A., Tennstedt, F. (1985): Der Weg zum »Gesetz über die Vereinheitlichung des Gesundheitswesen « vom 3. Juli 1934. Entwicklungslinien und -momente des staatlichen und kommunalen Gesundheitswesens in Deutschland (Schriftenreihe der Akademie für öffentliches Gesundheitswesen in Düsseldorf, Bd. 13), Düsseldorf

Lehmkuhl, Dr. (1939/40): Zur Technik der Erbbestandsaufnahme, in: Der öffentliche Gesundheitsdienst, Ausg. A, 5. Jg., S. 144 f.

Lenz, F. (1932): Menschliche Auslese und Rassenhygiene (Eugenik) (= Baur, E., Fischer, E., Lenz, F., Menschliche Erblichkeitslehre und Rassenhygiene, Bd. 2), 4. Aufl., München

Linden, H. (1935): Erb- und Rassenpflege bei den Gesundheitsämtern, in: Der öffentliche Gesundheitsdienst, Ausg. A, 1. Jg., S. 3-13

Linden, H. (1942): Die Eheunbedenklichkeitsbescheinigung. Bemerkungen zu dem vorstehenden Aufsatz von Stadtmedizinalrat Dr. Seeger, Königsberg, in: Der öffentliche Gesundheitsdienst, Ausg. B, 8. Jg., S. 239 f.

Macnicol, J. (1989): Eugenics and the Campaign for Voluntary Sterilization in Britain between the Wars, in: Social History of Medicine, Vol. 2, S. 147-169

Mitscherlich, A., Mielke, F. (1979): Medizin ohne Menschlichkeit. Dokumente des Nürnberger Ärzteprozesses, 91.-95. Tsd., Frankfurt a.M.

Müller-Freienfels, W. (1962): Ehe und Recht, Tübingen

Müller-Hill, B. (1984): Tödliche Wissenschaft. Die Aussonderung von Juden, Zigeunern und Geisteskranken 1939-1945, Reinbek

Reeg, K.P. (1988): Friedrich Georg Christian Bartels (1892-1968). Ein Beitrag zur Entwicklung der Leistungsmedizin im Nationalsozialismus (Abhandlungen zur Geschichte der Medizin und der Naturwissenschaften, Heft 56), Husum

Roth, K.H. (1984): »Erbbiologische Bestandsaufnahme«. Ein Aspekt »ausmerzender« Erfassung vor der Entfesselung des Zweiten Weltkrieges, in: Redaktionskollektiv Autonomie, Roth, K.H. (Hg.), Erfassung zur Vernichtung. Von der Sozialhygiene zum Gesetz über Sterbehilfe, S. 57-100

Roth, K.H. (1986): Schöner neuer Mensch. Der Paradigmenwechsel der klassischen Genetik und seine Auswirkungen auf die Bevölkerungsbiologie des »Dritten Reichs«, in: Kaupen-Haas, H. (Hg.), Der Griff nach der Bevölkerung. Aktualität und Kontinuität nazistischer Bevölkerungspolitik (Schriften der Hamburger Stiftung für Sozialgeschichte des 20. Jahrhunderts, Bd. 1), Nördlingen, S. 11-63

Strickberger, M.W.(1988): Genetik, deutschsprachige Ausgabe übersetzt von Schmieger, H. und Schmidt, C., München, Wien (3. Aufl. New York 1985)

Szasz, T.S. (1979): Schizophrenie - das heilige Symbol der Psychiatrie, Wien, München, Zürich (Originalausgabe: Schizophrenia, New York 1976, übers. von B. Stein)

Kallmann, F.J. (1938): The Genetics of Schizophrenia: A Study of Heredity and Reproduction in the Families of 1.087 Schizophrenics, New York

Usborne, C. (1989): The Politics of Fertility Control in Germany 1910-1927, Ph. D. Open University Milton Keynes

Vellguth, H. (1938/39): Ziel und Methoden der Erbbestandsaufnahme, in: Der öffentliche Gesundheitsdienst, Ausg. A, 4. Jg., S. 495-501

Verschuer, O. Freiherr von (1939): Vier Jahre Frankfurter Universitätsinstitut für Erbbiologie und Rassenhygiene, in: Der Erbarzt (Beilage zum Deutschen Ärzteblatt), 6. Jg., S. 57-63 
Weindling, P. (1984): Die preußische Medizinalverwaltung und die »Rassenhygiene «. Anmerkungen zur Gesundheitspolitik der Jahre 1905-1933, in: Zeitschrift für Sozialreform 30, S. 675-687

Weindling, P. (1985): The Kaiser Wilhelm Institute for Anthropology, Human Heredity and Eugenics in Social Context, in: Annals of Science, Vol. 42, S. 303-318

Weingart, P./Kroll, J./Bayertz, K. (1988): Rasse, Blut und Gene. Geschichte der Eugenik und Rassenhygiene in Deutschland, Frankfurt

Weß, L. (1989): Die Träume der Genetik. Gentechnische Utopien vom sozialen Fortschritt (Schriften der Hamburger Stiftung für Sozialgeschichte des 20. Jahrhunderts, Bd. 6), Hamburg 